\title{
L-carnitine supplementation in patients with HIVIAIDS and fatigue: a double-blind, placebo-controlled pilot study
}

This article was published in the following Dove Press journal:

HIVIAIDS - Research and Palliative Care

19 February 2015

Number of times this article has been viewed

\author{
Ricardo A Cruciani' \\ Manuel Revuelta ${ }^{2}$ \\ Ella Dvorkin ${ }^{3}$ \\ Peter $\mathrm{Homel}^{4}$ \\ Pauline Lesage ${ }^{5}$ \\ Nora Esteban-Cruciani ${ }^{6}$ \\ 'Center for Comprehensive Pain \\ Management and Palliative Care, \\ Capital Institute for Neurosciences, \\ Capital Health Medical Center, \\ Pennington, NJ, ${ }^{2}$ Lee Memorial \\ Hospital, Fort Myers, FL, ${ }^{3}$ Institutional \\ Review Board, New York University, \\ New York, NY, ${ }^{4}$ Department of Pain \\ Medicine and Palliative Care, Beth \\ Israel Medical Center, New York, \\ NY, ${ }^{5}$ Maimonides Medical Center, \\ Brooklyn, NY, ${ }^{6}$ Children's Hospital at \\ Montefiore, Albert Einstein College \\ of Medicine, Bronx, NY, USA
}

Background: The purpose of this study was to determine the effect of L-carnitine supplementation on fatigue in patients with terminal human immunodeficiency virus/acquired immune deficiency syndrome (HIV/AIDS).

Methods: In this randomized, double-blind, placebo-controlled, parallel-group study, patients who had end-stage HIV/AIDS with carnitine deficiency and fatigue received $3 \mathrm{~g}$ of oral L-carnitine or placebo for 2 weeks, followed by a 2-week, open-label phase with the same amount of L-carnitine for all patients. The primary outcome was the degree of fatigue according to the Brief Fatigue Inventory. Secondary outcomes included serum carnitine and lactate levels, physical, emotional, social, and functional well-being, performance status, mood, and CD4 count.

Results: Eighteen patients in the treatment arm and 17 in the placebo arm completed the trial. At the end of the double-blind phase, total and free carnitine levels in the treatment arm rose from $28 \pm 9$ to $48 \pm 17 \mathrm{nM} / \mathrm{L}(P<0.001)$ and from $24 \pm 8$ to $40 \pm 13 \mathrm{nM} / \mathrm{L}(P<0.001)$ respectively, with no changes in the placebo arm. The primary outcome, ie, fatigue measured at the end of the blinded phase, did not improve. Secondary outcomes of function, quality of life, and mood did not show improvement either. The secondary outcome of serum lactate decreased from baseline in the treatment group $(1.45 \pm 0.76$ to $1.28 \pm 0.52 \mathrm{mmol} / \mathrm{L})$ and increased in the placebo group $(1.38 \pm 0.62$ to $1.84 \pm 0.74 \mathrm{mmol} / \mathrm{L} ; P<0.005)$.

Conclusion: Our study suggests that $3 \mathrm{~g}$ of oral L-carnitine supplementation for 2 weeks in terminally ill HIV/AIDS patients does not improve fatigue. This study might help to determine the dose and duration of treatment used in future clinical trials, as higher doses and/or longer periods of supplementation might be needed in order to detect an improvement. The reduction in serum lactate levels suggests a potential role for L-carnitine supplementation in patients undergoing certain types of antiretroviral therapy. This study contributes evidence-based data to the field of alternative and complementary medicine, a multibillion dollar industry in which controlled studies are not the norm.

Keywords: acquired immune deficiency syndrome, human immunodeficiency virus, fatigue, lactate, L-carnitine supplementation, carnitine deficiency, palliative care

\section{Introduction}

Fatigue is among the most common symptoms reported by individuals infected with human immunodeficiency virus (HIV), with a prevalence that varies by disease status and having rates close to $80 \%$ in patients with acquired immune deficiency syndrome (AIDS). ${ }^{1}$ Persistent fatigue may be multifactorial, including anemia, diseasemodifying therapies, centrally-acting drugs, metabolic disturbances, mood disorders, and micronutrient deficiencies including carnitine. ${ }^{2}$ This micronutrient facilitates the 
translocation of long-chain fatty acids into the mitochondrial matrix for the production of energy, and is also necessary for the elimination of acyl products that interfere with it. ${ }^{3}$ Carnitine deficiency has been reported in a number of conditions, including cancer, ${ }^{4}$ renal insufficiency, ${ }^{5}$ and liver insufficiency, ${ }^{3}$ and in pediatric ${ }^{6}$ and adult ${ }^{7}$ patients with HIV/ AIDS. However, little is known about the role of L-carnitine supplementation in the management of fatigue symptoms in patients with HIV/AIDS and carnitine deficiency. The present study was designed to evaluate the effect of L-carnitine supplementation on fatigue in patients with advanced HIV/ AIDS and carnitine deficiency.

\section{Materials and methods}

The aim of this randomized, double-blind, placebo-controlled, parallel-group study was to determine the effect of L-carnitine supplementation on fatigue in patients with end-stage HIV/ AIDS and serum carnitine deficiency. Other outcomes included carnitine blood levels, performance status, cognitive function, mood, quality of life, CD4 and CD8 count, and serum lactic acid levels. The protocol and consent form were approved by the institutional review board at Beth Israel Medical Center, New York, and all patients provided their signed informed consent prior to participation.

\section{Study population}

The study population included adult patients with advanced HIV/AIDS recruited from the HIV/AIDS clinic at Beth Israel Medical Center. Eligible patients had the following characteristics: reported fatigue of moderate to severe intensity, a Karnofsky Performance Status (KPS) score $^{8} \geq 50$, and car-

Table I Demographic and laboratory characteristics in the placebo and L-carnitine supplementation arms

\begin{tabular}{|c|c|c|c|}
\hline Characteristics & $\begin{array}{l}\text { Placebo } \\
(n=17)\end{array}$ & $\begin{array}{l}\text { L-carnitine } \\
(n=18)\end{array}$ & $P$-value* \\
\hline Age, years & $45.5 \pm 5.4$ & $48.9 \pm 8.8$ & 0.21 \\
\hline Female & $3(18 \%)$ & I (6\%) & 0.26 \\
\hline \multicolumn{4}{|l|}{ Race } \\
\hline Black & $2(12 \%)$ & $3(17 \%)$ & 0.31 \\
\hline Hispanic & $5(29 \%)$ & $9(50 \%)$ & \\
\hline White & $10(59 \%)$ & $6(33 \%)$ & \\
\hline KPS score & $75.0 \pm 6.3$ & $71.9 \pm 6.6$ & 0.17 \\
\hline Lactate (mmol/L) & $1.3 \pm 0.6$ & $1.5 \pm 0.8$ & 0.41 \\
\hline Hemoglobin (mmol/L) & $14.6 \pm 1.4$ & $13.5 \pm 1.8$ & 0.05 \\
\hline BUN (mmol/L) & $13.6 \pm 3.4$ & $16.3 \pm 5.0$ & 0.07 \\
\hline Creatinine (mmol/L) & $0.9 \pm 0.2$ & $1.0 \pm 0.2$ & 0.15 \\
\hline CD4 & $370(62,87 I)^{d}$ & $299(95,1000)^{d}$ & 0.32 \\
\hline
\end{tabular}

Notes: The data are shown as the mean \pm standard deviation; $* P$-value comparing the placebo and L-carnitine supplementation arms at baseline. ${ }^{d}=$ range.

Abbreviations: BUN, blood urea nitrogen; KPS, Karnofsky Performance Status. nitine deficiency (Table 1). Carnitine deficiency was defined as free carnitine $<35 \mathrm{nM} / \mathrm{L}$ for males and $<25 \mathrm{nM} / \mathrm{L}$ for females (normal range 35-67 and 25-55, respectively; Quest Diagnostics, San Diego, CA, USA) ${ }^{9}$ or an acyl-carnitine ratio $>0.4$ acyl-carnitine ratio ([total acyl-carnitine minus free acyl-carnitine)/free acyl-carnitine]). ${ }^{9,10}$ Exclusion criteria included: uncontrolled or severe cardiovascular, pulmonary, or renal disease; psychiatric disorder sufficient to compromise adherence to study methods or data collection; history of stroke, seizure, or primary or metastatic brain tumor; history of dementia, aphasia, or other deficits of cognition or speech/language; known allergy to carnitine; therapy with any form of carnitine $<12$ months prior to enrollment; pregnancy or breast feeding; any acute illness within 30 days before study entry; and active drug/alcohol use or dependence. Patients were treated with combinations of the following medications (n): amprenavir (3), indinavir (2), delavirdine (2), emtricitabine (2), lamivudine (9), abacavir + lamivudine (2), enfuvirtide (2), saquinavir (2), lopinavir + ritonavir (11), fosamprenavir (1), nevirapine (6), ritonavir (10), atazanavir sulfate (6), abacavir plus zidovudine plus lamivudine (5), tenofovir plus emtricitabine (2), didanosine (2), tenofovir disoproxil fumarate (19), nelfinavir (4), stavudine (3), and abacavir sulfate (6).

\section{Procedures}

Eligible patients who provided signed informed consent underwent a medical history and physical examination, as well as blood sampling for plasma carnitine determination. After the samples were collected, the tubes were wrapped in alumina foil to prevent degradation of carnitine by exposure to light and shipped on ice to the laboratory the same day. Plasma carnitine levels were measured using ultra performance liquid chromatography tandem mass spectrometry. Plasma $50 \mu \mathrm{L}$ was spiked with an internal standard mixture containing D9-carnitine. After precipitation of the proteins with acetonitrile/methanol (2:1), the supernatant was evaporated to dryness and resuspended in $20 \%$ methanol. Carnitine was separated using a water/methanol gradient containing $10 \mathrm{mmol} / \mathrm{L}$ heptafluorobutyric acid and $10 \mathrm{mmol} / \mathrm{L}$ ammonium acetate. Carnitine was quantified by positive electrospray ionization in the multiple-reaction monitoring mode relative to an external standard curve (Quest Diagnostics). ${ }^{9}$ Patients who had carnitine deficiency underwent additional laboratory screening, including a complete blood count with differential and platelet count, creatinine, electrolytes, albumin, creatine phosphokinase, lactic acid, liver and renal function tests, prothrombin time/partial thromboplastin time, 
thyroid function tests, total iron binding content, ferritin levels, vitamin B12 and folate levels, CD4 count, viral load, hepatitis $\mathrm{C}$ antibodies, and testosterone, and a pregnancy test if indicated.

Carnitine-deficient patients who met the eligibility criteria completed the questionnaire packet (see below) and were then randomized into treatment groups using a standard computer program. Randomization included stratification for hemoglobin level (below/above $10 \mathrm{~g} / \mathrm{dL}$ ) to account for the prominence of anemia-related fatigue (Figure 1).

Patients were provided with the study drug at a concentration of $1 \mathrm{~g}$ of L-carnitine $/ 10 \mathrm{~mL}$ or equivalent placebo (made of the same syrup in which L-carnitine was dissolved for the treatment

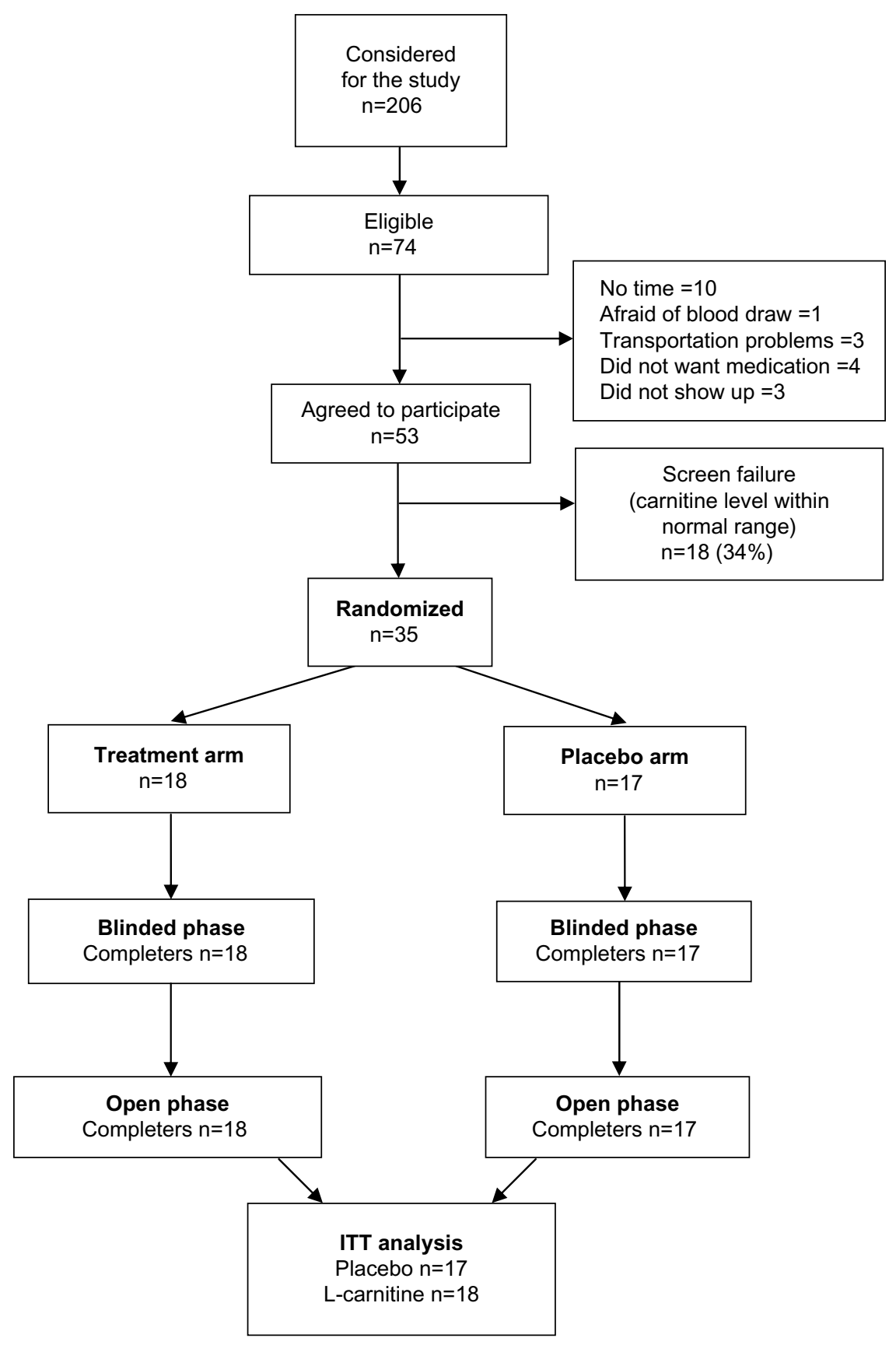

Figure I Patient disposition. Patients were screen for inclusion and exclusion criteria and randomized into two arms, ie, sham and L-carnitine supplementation. Patients were stratified by hemoglobin level.

Abbreviation: ITT, intent-to-treat. 
arm), and instructions on storage conditions, preservation, and disposition of any unused drug. The liquid L-carnitine and placebo were prepared by a research pharmacist and were identical in appearance and taste. Coded dark-brown opaque bottles were dispensed to the patients, and neither the patient nor the investigator knew the identity of the material dispensed. The initial dose was $0.5 \mathrm{~g}$ /day of L-carnitine or placebo once a day for 2 days. The dose was then increased to $0.5 \mathrm{~g}$ twice a day for 2 days. The patients were instructed to take $1.0 \mathrm{~g}$ twice a day for 2 days and finally to increase to $1.5 \mathrm{~g}$ twice daily for the remaining 8 days.

This blinded phase was followed by an open-label phase, during which all patients received L-carnitine supplementation for 2 weeks. Patients who had received placebo underwent a titration similar to the carnitine arm in the double-blind phase. Patients in the carnitine group continued on the same final dose taken during the randomized phase of the study. In addition to the baseline visit, patients were evaluated in person by an investigator at the end of the blinded phase and at the end of the 2-week open-label phase. The self-report measures were completed at each patient visit, including baseline, at the end of the blinded phase, and at the end of the open-label phase. During the blinded phase of the study, each patient was also contacted by telephone at least every other day.

\section{Outcomes}

The measures comprised validated instruments that assessed fatigue and other outcomes, as follows:

\section{Brief Fatigue Inventory}

The Brief Fatigue Inventory $(\mathrm{BFI})^{11}$ is a ten-item scale that assesses severity of fatigue (worst, usual, and right now) and also its interference with activities of daily living. A global fatigue score can be obtained by averaging all the items on the BFI. Estimates of reliability based on Cronbach's alpha ranged from 0.82 to 0.97 .

\section{Linear Analog Scale-Anemia}

The Linear Analog Scale-Anemia (LASA) ${ }^{12}$ includes three separate $100 \mathrm{~mm}$ visual analog scales that measure energy level, activity level, and overall quality of life. Internal consistency of the three items together is 0.97 . Higher scores represent better performance.

\section{Functional Assessment Cancer Therapy}

The Functional Assessment Cancer Therapy (FACT) ${ }^{13}$ is a multidimensional multi-item quality of life instrument based on the family of Functional Assessment of Chronic Illness Treatment scales. A higher score is indicative of better quality of life. Subscores are calculated as the sum of the responses to questions related to the domains of physical well-being (ten items), emotional well-being (ten items), social wellbeing (eight items), functional well-being (13 items), and cognitive functioning (three items). These can be summed to form a total score. The overall alpha measure of internal consistency is 0.91 , while alphas for subscores range between 0.73 (social well-being) and 0.91 (physical well-being).

\section{Mini Mental Status Examination}

The Mini Mental Status Examination ${ }^{14}$ is a brief test of memory, spatial ability, language comprehension, and reasoning. Test-retest reliability is 0.89 . Higher scores represent better mental functioning.

\section{Karnofsky Performance Status}

The $\mathrm{KPS}^{8}$ is a well-known, observer-rated measure of overall performance status. It has been shown to have good inter-rater reliability in the HIV population.

\section{Memorial Symptom Assessment Scale-Short Form}

The Memorial Symptom Assessment Scale-Short Form (MSAS-SF) ${ }^{15}$ assesses 32 physical and psychological symptoms occurring during the previous week. Separate questions ask about the frequency with which symptoms occur and the distress that the patient experiences in relation to that symptom. A total score can be calculated, as well as subtests for physical and psychological distress and a general symptom distress score. Cronbach's alpha estimates of reliability range between 0.76 and 0.87 for the subtests, and one-day testretest reliabilities range between 0.86 and 0.94 .

\section{Clinical Evaluation Scale of Depression}

The Clinical Evaluation Scale of Depression (CES-D) ${ }^{16}$ is a 20-item self-report scale intended to measure depressive symptoms experienced in the past week. Patients are asked to say how often they experienced each symptom, from less than a day to most or all of the time (5-7 days). Higher scores are indicative of greater degrees of depression, and a score of 16 or higher is indicative of clinically significant depression. The Cronbach's alpha is reported as 0.85 .

\section{Adverse effects}

At each contact, the patient was asked questions about side effects or other problems. If the patient reported any side effect associated with treatment, additional information 
was obtained, including onset, course, severity, and relationship to the drug. No patient required discontinuation of supplementation.

\section{Statistical analysis}

Sample size calculations based on earlier controlled trials ${ }^{4,17,18}$ suggested that 20 patients per group would be required to detect a 0.8 standard deviation difference in fatigue between groups, as measured by the BFI worst fatigue scale. Descriptive statistics are presented as the mean \pm standard deviation for normally distributed continuous variables (eg, age), the median (minimum, maximum) for skewed continuous variables (eg, CD4), and as the frequency (\%) for categorical variables (eg, race). The primary analysis was done using mixed model linear regression analysis comparing baseline worst fatigue on the BFI with worst fatigue after being randomized to either L-carnitine or placebo. Secondary analyses using mixed model regression were done to look at other outcomes possibly related to fatigue, such as quality of life as measured by the FACT. Efficacy analyses were done on an intent-to-treat basis. Mixed model regression was used to compare changes in outcome from baseline (week 0) to the end of the blinded phase (week 2) and from the end of the blinded phase to the end of the open-label phase. Mean contrast tests within the mixed model regression analysis were used to obtain estimates of the $P$-values for this comparisons. ${ }^{19}$

\section{Results}

Due to slow patient accrual, the study was terminated before full enrollment was achieved. There was a total of 17 patients enrolled in the placebo group and 18 patients in the L-carnitine group (Figure 1). These patients constituted the samples used for the intent-to-treat analysis.

\section{Carnitine levels}

Of the 53 patients who underwent blood carnitine determinations, 35 had levels below the normal range, suggesting a prevalence of $66 \%$ carnitine deficiency in this patient population. As shown in Table 1, the placebo and L-carnitine arms were similar in terms of demographic and clinical characteristics. Age was not significantly different between the groups $(P=0.20)$. Females comprised $18 \%$ of the placebo group versus $6 \%$ in the L-carnitine group, although this difference was not significant $(P=0.3)$. The race and ethnicity distribution between the groups was not proportionate, but again this difference was not significant $(P=0.31)$.

\section{Double-blind phase}

The results of the intent-to-treat analyses are shown in Table 2. The primary outcome of fatigue measured by change in scores on the BFI, ie, worst fatigue, fatigue now, and usual fatigue, did not show significant differences between the L-carnitine supplementation arm and the placebo arm ( $P=0.22, P=0.31$, and $P=0.35$, respectively). The secondary outcomes that showed an improvement in scores were the MSAS-SF physical distress scale, the MSAS-SF psychological distress scale, and the FACT cognitive functioning scale ( $P<0.08, P<0.05$, and $P<0.06$, respectively). All laboratory tests were similar in the two arms, except for a statistically significant lower lactate level in favor of the L-carnitine supplementation arm $(1.38 \pm 0.62 \mathrm{nmol} / \mathrm{L}$ and $1.84 \pm 0.74 \mathrm{nmol} / \mathrm{L}$ at baseline and at the end of the first phase for the placebo arm, and $1.45 \pm 0.76 \mathrm{nmol} / \mathrm{L}$ and $1.28 \pm 0.52 \mathrm{nmol} / \mathrm{L}$, respectively, for the L-carnitine arm, $P<0.005$, Table 2). None of the other outcomes (KPS, LASA, and CES-D) were significantly different between the groups.

\section{Open-label phase}

During the open-label phase, patients in both arms received $3 \mathrm{~g}$ of L-carnitine supplementation in the same fashion as for the patients in the L-carnitine supplementation arm in the first phase (Table 3). A comparison of serum carnitine levels between the end of the closed-label phase and the end of the open-label phase showed that the L-carnitine group remained steady albeit at a high level, while the placebo first group showed increased carnitine levels and narrowed the gap with the L-carnitine first group $(P<0.32$ and $P<0.31$ for free and total L-carnitine, respectively). The primary outcome and all secondary outcomes were similar in both arms, except for BFI usual fatigue scores, which were significantly lower in the L-carnitine first treatment arm $(P<0.05$, Table 3). Overall, L-carnitine supplementation was well tolerated, as shown in Table 4.

\section{Discussion}

This is a randomized, double-blind clinical trial in HIV/ AIDS patients with carnitine deficiency, in whom 2 weeks of L-carnitine supplementation at $3 \mathrm{~g}$ daily did not achieve an improvement in the primary outcome measure of fatigue. Secondary outcomes measures aimed to assess function, quality of life, and mood, and did not show improvement except for the MSAS psychological distress scale $(P<0.05)$ and a trend towards improvement in the FACT total score $(P<0.06)$ and the FACT cognitive functioning score $(P<0.07)$ that was not statistically significant. 
Table 2 Comparison of carnitine levels, lactate levels, and primary and secondary outcomes between baseline and the end of the placebo-controlled phase: results of mixed model regression based on intent-to-treat

\begin{tabular}{|c|c|c|c|c|c|}
\hline \multirow[t]{2}{*}{ Outcome } & \multicolumn{2}{|l|}{ Placebo } & \multicolumn{2}{|c|}{ L-Carnitine } & \multirow[t]{2}{*}{$P$-value* } \\
\hline & Baseline & Randomized & Baseline & Randomized & \\
\hline Total carnitine & $28.4 \pm 5.0$ & $29.4 \pm 6.1$ & $28.2 \pm 9.1$ & $47.9 \pm 17.1$ & $<0.001$ \\
\hline Free carnitine & $24.4 \pm 4$ & $24.2 \pm 5.0$ & $24.3 \pm 7.6$ & $40.58 \pm 13.7$ & $<0.001$ \\
\hline Acyl-carnitine ratio & $0.16 \pm 5$ & $0.21 \pm 6$ & $0.16 \pm 8$ & $0.18 \pm 15$ & 0.44 \\
\hline Lactate & $1.38 \pm 0.62$ & $\mathrm{I} .84 \pm 0.74$ & $1.45 \pm 0.76$ & $1.28 \pm 0.52$ & $<0.005$ \\
\hline Worst fatigue & $6.6 \pm 2.4$ & $5.9 \pm 2.7$ & $7.1 \pm 2.0$ & $7.2 \pm 1.7$ & 0.22 \\
\hline Fatigue now & $6.1 \pm 1.5$ & $4.8 \pm 2.2$ & $6.3 \pm 1.6$ & $5.6 \pm 1.6$ & 0.31 \\
\hline Usual fatigue & $5.8 \pm 2.0$ & $5.1 \pm 1.8$ & $6.3 \pm 1.7$ & $6.3 \pm 1.9$ & 0.35 \\
\hline Total BFI & $6.3 \pm 1.6$ & $5.0 \pm 2.4$ & $6.9 \pm 1.7$ & $6.1 \pm 1.5$ & 0.33 \\
\hline LASA energy & $37.1 \pm 15.7$ & $50.5 \pm 22.0$ & $32.6 \pm 16.1$ & $47.0 \pm 20.1$ & 0.97 \\
\hline LASA daily & $42.6 \pm 14.9$ & $50.7 \pm 19.4$ & $40.4 \pm 22.1$ & $54.6 \pm 20.1$ & 0.69 \\
\hline LASA QoL & $46.6 \pm 15.5$ & $54.9 \pm 20.2$ & $43.0 \pm 23.6$ & $54.1 \pm 20.9$ & 0.84 \\
\hline MSAS Phys & $1.3 \pm 0.5$ & $1.2 \pm 0.7$ & I. $8 \pm 0.7$ & $1.3 \pm 0.5$ & 0.08 \\
\hline MSAS Psych & $1.8 \pm 1.0$ & $1.9 \pm 1.0$ & $2.3 \pm 0.6$ & $1.8 \pm 0.7$ & 0.05 \\
\hline MSAS GDI & $2.0 \pm 0.7$ & $1.7 \pm 0.9$ & $2.2 \pm 0.5$ & $1.7 \pm 0.6$ & 0.47 \\
\hline CES-D & $23.2 \pm 12.1$ & $21.1 \pm 13.01$ & $24.8 \pm 9.8$ & $16.4 \pm 6.9$ & 0.16 \\
\hline FACT total & $68.7 \pm 11.4$ & $62.6 \pm 11.4$ & $66.8 \pm 9.7$ & $64.8 \pm 10.2$ & 0.06 \\
\hline FACT PWB & $\mid 4.7 \pm 5.6$ & $11.0 \pm 6.8$ & $16.5 \pm 6.1$ & $12.3 \pm 5.4$ & 0.46 \\
\hline FACT EWB & $12.6 \pm 6.4$ & $10.4 \pm 7.2$ & $13.2 \pm 7.2$ & $10.6 \pm 7.3$ & 0.78 \\
\hline FACT FWB & $16.3 \pm 6.5$ & $15.4 \pm 6.6$ & $13.9 \pm 4.4$ & $15.4 \pm 2.5$ & 0.10 \\
\hline FACT SWB & $18.1 \pm 7.6$ & $19.4 \pm 7.7$ & $17.7 \pm 7.6$ & $20.1 \pm 6.6$ & 0.64 \\
\hline FACT CF & $7.2 \pm 2.2$ & $6.3 \pm 2.6$ & $6.0 \pm 3.1$ & $6.5 \pm 2.6$ & 0.07 \\
\hline MMSE & $29.6 \pm 1.1$ & $29.7 \pm 0.8$ & $29.0 \pm 1.3$ & $29.6 \pm 0.8$ & 0.25 \\
\hline
\end{tabular}

Notes: The data are shown as the mean \pm standard deviation; ${ }^{* P}$-value comparing the placebo and L-carnitine supplementation arms at baseline and at the end of the closedlabel phase.

Abbreviations: BFI, Brief Fatigue inventory; LASA, Linear Analog Scale-Anemia; MSAS, Memorial Symptom Assessment Scale; GDI, Global Distress Index; Psych, psychological distress; Phys, physical distress; CES-D, Clinical Evaluation Scale of Depression; FACT, Functional Assessment Cancer Therapy; CF, cognitive functioning; PWB, physical wellbeing; SWB, social well-being; EWB, emotional well-being; FWB, functional well-being; MMSE, Mini Mental Status Examination; QoL, quality of life.

These results are in conflict with previous reports of improvement in symptoms of fatigue in patients with cancer $^{4,14,15}$ or hepatic disease ${ }^{20,21}$ and in elderly patients. ${ }^{22}$ However, two recent large clinical trials in cancer patients also showed no improvement in fatigue after L-carnitine supplementation. ${ }^{23,24}$ Possible reasons for this negative primary outcome include differences in study population, duration of treatment, dose of L-carnitine, noncompliance with study medication, and coadministration of other supplements. The present trial was conducted in patients with HIV/AIDS who were approaching end of life, with $83 \%$ having a life expectancy of less than a month, while most of the studies showing improvement in fatigue were conducted in more stable patients. The duration of carnitine supplementation varied significantly between studies (ranging from 2 to 16 weeks), and the 2 weeks of supplementation in this study might not have been sufficient to detect an effect. The dose of L-carnitine also varied among the studies (2-6 $\mathrm{g}$ a day), and higher doses than the one used in this study ( $3 \mathrm{~g} /$ day) might be needed to achieve relief of fatigue. ${ }^{25}$ In the absence of definitive studies on HIV/AIDS patients at the time this study was developed, we selected our dose of L-carnitine from a dose-finding, open-label trial ${ }^{5}$ and a small controlled clinical trial, ${ }^{18}$ both conducted by our group, and from an open-label study by Graziano et $\mathrm{al}^{17}$ who suggested that $2-3 \mathrm{~g}$ of daily oral L-carnitine supplementation was adequate to improve fatigue in cancer patients. The studies showing improvement of fatigue with 4-6 g/day for 12 weeks were not available when we embarked on the present study. ${ }^{25,26}$ Inadequate intake of the study drug, hence failure to increase plasma carnitine levels by the end of the supplementation phase, could have been another potential contributor to the lack of improvement in fatigue. However, in the present study, 2 weeks of oral L-carnitine supplementation at $3 \mathrm{~g}$ /day resulted in higher plasma carnitine levels $(P<0.001)$, which were similar to those at the end of the open-label phase after 2 additional weeks of supplementation. Interestingly, the acyl-carnitine ratio did not change significantly in the treatment arm after supplementation, suggesting that it might not be a reliable indicator of deficiency. Also, carnitine alone might not be sufficient to improve fatigue, as suggested in the 
Table 3 Comparison of carnitine levels and primary and secondary outcomes between the end of the placebo phase and the end of the open-label phase

\begin{tabular}{llll}
\hline Outcome & Placebo first & L-carnitine first & $P$-value* \\
\hline Total L-carnitine & $43.1 \pm 11.3$ & $47.9 \pm 16.0$ & 0.32 \\
Free L-carnitine & $36.0 \pm .9 .7$ & $40.2 \pm 14.0$ & 0.31 \\
Total BFI & $3.5 \pm 2.4$ & $3.5 \pm 2.2$ & 0.15 \\
Fatigue now & $3.2 \pm 2.2$ & $3.5 \pm 2.1$ & 0.50 \\
Worst fatigue & $4.1 \pm 2.4$ & $4.1 \pm 2.4$ & 0.19 \\
Usual fatigue & $3.7 \pm 2.6$ & $3.0 \pm 1.8$ & 0.05 \\
LASA energy & $63.6 \pm 22.9$ & $61.1 \pm 14.5$ & 0.87 \\
LASA daily & $66.0 \pm 21.1$ & $66.4 \pm 16.4$ & 0.61 \\
LASA QoL & $64.1 \pm 21.1$ & $70.3 \pm 19.3$ & 0.24 \\
MSAS Phys & $1.1 \pm 0.6$ & $1.3 \pm 0.8$ & 0.39 \\
MSAS Psych & $1.7 \pm 1.0$ & $1.8 \pm 0.8$ & 0.54 \\
MSAS GDI & $1.5 \pm 0.9$ & $1.6 \pm 0.7$ & 0.74 \\
CES-D & $18.2 \pm 13.0$ & $16.3 \pm 11.8$ & 0.43 \\
FACT total & $63.6 \pm 9.2$ & $61.8 \pm 7.2$ & 0.23 \\
FACT PWB & $8.7 \pm 6.9$ & $9.4 \pm 5.9$ & 0.45 \\
FACT EWB & $10.0 \pm 7.4$ & $9.1 \pm 6.4$ & 0.51 \\
FACT FWB & $16.9 \pm 5.8$ & $16.8 \pm 4.2$ & 0.91 \\
FACT SWB & $20.3 \pm 6.8$ & $19.4 \pm 6.9$ & 0.94 \\
FACT CF & $7.7 \pm 2.4$ & $7.2 . \pm 3.0$ & 0.23 \\
MMSE & $29.7 \pm 0.6$ & $29.4 \pm 1.0$ & 0.27 \\
\hline NAS Dat &
\end{tabular}

Notes: Data are shown as the mean \pm standard deviation; ${ }^{*} P$-value comparing the end of closed-label phase with the end of the open-label phase.

Abbreviations: BFI, Brief Fatigue inventory; LASA, Linear Analog Scale-Anemia; MSAS, Memorial Symptom Assessment Scale; GDI, Global Distress Index; Psych, psychological distress; Phys, physical distress; CES-D, Clinical Evaluation Scale of Depression; FACT, Functional Assessment Cancer Therapy; CF, cognitive functioning; PWB, physical well-being; SWB, social well-being; EWB, emotional well-being; FWB, functional well-being; MMSE, Mini Mental Status Examination QoL, quality of life.

five-arm randomized controlled trial by Mantovani et al, who reported improved fatigue scores in patients receiving a combination of L-carnitine $4 \mathrm{~g}$ /day plus eicosapentaenoic acid, medroxyprogesterone, and thalidomide, but not with carnitine alone. ${ }^{26}$

Table 4 Comparison of side effects between placebo and L-carnitine supplementation arm by the end of the placebocontrolled phase $(P>0.05)$

\begin{tabular}{lll}
\hline Adverse events & $\begin{array}{l}\text { Placebo } \\
(\mathbf{n}=17)\end{array}$ & $\begin{array}{l}\text { L-carnitine } \\
(\mathbf{n}=18)\end{array}$ \\
\hline Cold symptoms & $\mathrm{I}(6 \%)$ & $2(\mathrm{II} \%)$ \\
Diarrhea & $2(12 \%)$ & $2(\mathrm{II} \%)$ \\
Dizziness & $2(12 \%)$ & $0(0 \%)$ \\
Headache & $3(18 \%)$ & $\mathrm{I}(6 \%)$ \\
Hoarseness & $\mathrm{I}(6 \%)$ & $\mathrm{I}(6 \%)$ \\
Nervousness & $2(12 \%)$ & $0(0 \%)$ \\
Sore throat & $\mathrm{I}(6 \%)$ & $\mathrm{I}(6 \%)$ \\
Stomach discomfort & $\mathrm{I}(6 \%)$ & $2(11 \%)$ \\
Other adverse events* & 10 & 12 \\
\hline
\end{tabular}

Note: *Sum of all events, not independent count.
In this study, we did not detect an increase in CD4 and CD8 counts as reported by Moretti et al, ${ }^{25}$ possibly due to differences in study design. Ours was a randomized controlled trial, the duration of L-carnitine supplementation was 2 weeks, and the dose of oral L-carnitine was $3 \mathrm{~g} /$ day, while the study by Moretti et al was open-label, the duration of supplementation was 16 weeks, and $6 \mathrm{~g}$ /day of L-carnitine was administered intravenously. ${ }^{25}$

An interesting observation is the decrease in lactate levels associated with L-carnitine supplementation in our study, because type B lactic acidosis has been reported in $8 \%-35 \%$ of HIV patients treated with nucleoside reverse transcriptase inhibitors. ${ }^{27}$ Twenty-three of the patients in this study (eleven in the control arm and 12 in the treatment arm) were treated with ritonavir alone or in combination with lopinavir, a drug that has been reported to cause lactic acidosis in $2 \%$ of cases. ${ }^{28}$ Nineteen patients were on tenofovir disoproxil fumarate (a first-line drug for the treatment of chronic hepatitis B) ${ }^{29}$ which is also reported to cause lactic acidosis. Overall, lactic acidosis is usually mild (serum lactate $<2 \mathrm{mmol} / \mathrm{L}$ ), but can progress to severe $(>5 \mathrm{mmol} / \mathrm{L})$. In addition, stavudine and didanosine, two drugs that are rarely used as main therapy, sometimes prescribed in combination with highly active antiretroviral therapy, can produce acute life-threatening lactic acidosis. ${ }^{28}$ Several strategies have been proposed for the treatment of lactic acidosis. Case reports have suggested a role for thiamine $(100 \mathrm{mg})$ and riboflavin $(50 \mathrm{mg}) \cdot{ }^{30}$ Also, in a small case series involving six AIDS patients with severe lactic acidosis $(>9 \mathrm{~nm} / \mathrm{L})$, it was observed that lactate levels normalized after supplementation with acetyl-L-carnitine $1,000 \mathrm{mg}$ twice daily ${ }^{31}$ Our observation of a decrease in lactate by the end of the blinded phase should be interpreted with caution due to the higher levels of lactate observed in the placebo arm, and the lack of data at the end of the double-blind phase.

This negative study has several important limitations. The study was powered for an effect size of $50 \%$, but more recent data suggest that an effect size of $30 \%$ would have been more appropriate, and as a result, our study might have been underpowered. In addition, the study had to be closed prematurely due to slow accrual. Patients were not enthusiastic about a study designed to control symptoms as oppose to cure, and they feared blood drawing would worsen fatigue. Another limitation is the number of outcome measures, which might have been excessive for the number of patients. However, due to the exploratory nature of this study, where the objective was to detect trends rather than definite values, we believe that it is permissible.

The results of this study do not support the use of L-carnitine supplementation to treat fatigue in HIV/AIDS. The 
potential beneficial effect of L-carnitine supplementation for symptoms of fatigue as detected by the significant improvement in the secondary outcome MSAS psychological distress scale $(P<0.05)$ and a trend towards improvement in the FACT total score $(P<0.06)$ and the FACT cognitive functioning score $(P<0.07)$, albeit not statistically significant, is of interest, but the study was not powered for these outcomes, and in view of a lack of effect on the primary outcome (fatigue symptoms as measured by the BFI), these could be spurious findings, so further studies are needed to verify our results.

This study provides data on the prevalence of carnitine deficiency in terminally ill patients with HIV/AIDS, and may assist in the selection of dose and treatment duration for future studies. The significant reduction in serum lactate levels by L-carnitine supplementation may be of clinical relevance for patients on certain antiretroviral agents and requires further investigation.

\section{Acknowledgment}

This work was funded in part by a grant from the National Institutes of Health (1-R21NR08295-0).

\section{Disclosure}

The authors report no conflicts of interest in this work.

\section{References}

1. Schifitto G, Deng L, Yeh TM, et al. Clinical, laboratory, and neuroimaging characteristics of fatigue in HIV-infected individuals. J Neurovirol. 2011;17:17-25.

2. Silvério R, Laviano A, Rossi Fanelli F, Seelaender M. L-carnitine and cancer cachexia: clinical and experimental aspects. $J$ Cachexia Sarcopenia Muscle. 2011;2:37-44.

3. Bonafé L, Berger MM, Que YA, Mechanick JI. Carnitine deficiency in chronic critical illness. Curr Opin Clin Nutr Metab Care. 2014;17: 200-209.

4. Cruciani RA, Dvorkin E, Homel P, et al. L-carnitine supplementation for the treatment of fatigue and depressed mood in cancer patients with carnitine deficiency: a preliminary analysis. Ann N Y Acad Sci. 2004;1033:168-176.

5. Yang SK, Xiao L, Song PA, Xu X, Liu FY, Sun L. Effect of L-carnitine therapy on patients in maintenance hemodialysis: a systematic review and meta-analysis. J Nephrol. 2014;27:317-329.

6. Esteban-Cruciani NV. Severe carnitine deficiency in children with AIDS: improved functional activity status after supplementation. Pediatr Res. 2001;49:254A.

7. De Simone C, Tzantzoglou S, Jirillo E, Marzo A, Vullo V, Martelli EA. L-carnitine deficiency in AIDS patients. AIDS. 1992;6:203-205.

8. Yates JW, Chalmer B, McKegney FP. Evaluation of patients with advanced cancer using the Karnofsky Performance Status. Cancer. 1980;40:2220-2224.

9. Quest Diagnostics [webpage on the Internet]. Available from http://www.questdiagnostics.com/testcenter/BUOrderInfo. action?tc=70107X\&labCode=SJC. Accessed 23 December 2014.

10. Belay B, Esteban-Cruciani N, Walsh CA, Kaskel FJ. The use of levocarnitine in children with renal disease: a review and a call for future studies. Pediatr Nephrol. 2006;21:308-317.
11. Mendoza TR, Wang XS, Cleeland CS, et al. The rapid assessment of fatigue severity in cancer patients: use of the Brief Fatigue Inventory. Cancer. 1999;85:1186-1196.

12. McCorkle R, Young K. Development of a symptom distress scale. Cancer Nurs. 1978;1:373-378.

13. Yellen SB, Cella DF, Webster K, Blendowski C, Kaplan E. Measuring fatigue and other anemia-related symptoms with the Functional Assessment of Cancer Therapy (FACT) measurement system. J Pain Symptom Manage. 1997;13:63-74.

14. Folstein MF, Folstein SE, McHugh PR. "Mini-mental state". A practical method for grading the cognitive state of patients for the clinician. J Psychiatr Res. 1975;12:189-198.

15. Chang VT, Hwang SS, Feuerman M, Kasimis BS, Thaler HT. The Memorial Symptom Assessment Scale Short Form (MSAS-SF). Cancer. 2000;89:1162-1171.

16. Radloff LS. The CES-D scale: a self-report depression scale for research in the general population. Appl Psychol Meas. 1977;1:385-401.

17. Graziano F, Bisonni R, Catalano V, et al. Potential role of levocarnitine supplementation for the treatment of chemotherapy-induced fatigue in non-anemic cancer patients. Br J Cancer. 2002;86:1854-1857.

18. Cruciani RA, Dvorkin E, Homel P, et al. L-carnitine supplementation in patients with advanced cancer and carnitine deficiency: a double-blind, placebo-controlled study. J Pain Symptom Manage. 2009;37:622-631.

19. Hedeker D, Gibbons RD. Application of random-effects pattern-mixture models for missing data in longitudinal studies. Psychol Methods. 1997;2:64-78.

20. Malaguarnera M, Gargante MP, Cristaldi E, et al. Acetyl-L-carnitine treatment in minimal hepatic encephalopathy. Dig Dis Sci. 2008;53: 3018-3025.

21. Malaguarnera M, Vacante M, Giordano M, et al. L-carnitine supplementation improves hematological pattern in patients affected by $\mathrm{HCV}$ treated with Peg interferon- $\alpha 2 \mathrm{~b}$ plus ribavirin. World J Gastroenterol. 2011;17:4414-4420.

22. Malaguarnera M, Cammalleri L, Gargante MP, Vacante M, Colonna V, Motta M. L-Carnitine treatment reduces severity of physical and mental fatigue and increases cognitive functions in centenarians: a randomized and controlled clinical trial. Am J Clin Nutr. 2007;86:1738-1744.

23. Cruciani RA, Zhang JJ, Manola J, Cella D, Ansari B, Fisch MJ. L-carnitine supplementation for the management of fatigue in patients with cancer: an Eastern Cooperative Oncology Group Phase III, randomized, double-blind, placebo-controlled trial. J Clin Oncol. 2012;30: 3864-3869.

24. Hershman DL, Unger JM, Crew KD, et al. Randomized double-blind placebo-controlled trial of acetyl-L-carnitine for the prevention of taxane-induced neuropathy in women undergoing adjuvant breast cancer therapy. J Clin Oncol. 2013;31:2627-2633.

25. Moretti S, Alesse E, Di Marzio L. Effect of L-carnitine on human immunodeficiency virus-1 infection-associated apoptosis: a pilot study. Blood. 1998;15;91:3817-3824.

26. Mantovani G, Macciò A, Madeddu C, et al. Randomized phase III clinical trial of five different arms of treatment in 332 patients with cancer cachexia. Oncologist. 2010;15:200-211.

27. Claessens YE, Chiche JD, Mira JP, Cariou A. Bench to bedside review: severe lactic acidosis in HIV patients treated with nucleoside analogue reverse transcriptase inhibitors. Crit Care. 2003;7:226-232.

28. Dlamini J, Ledwaba L, Mokwena N, et al. Lactic acidosis and symptomatic hyperlactataemia in a randomized trial of first-line therapy in HIV-infected adults in South Africa. Antivir Ther. 2011;16:605-609.

29. Ridruejo E, Silva MO. Safety of long-term nucleos(t)ide treatment in chronic hepatitis B. Expert Opin Drug Saf. 2012;11:357-360.

30. Fouty B, Frerman F, Reves R. Riboflavin to treat nucleoside analogues induced lactic acidosis. Lancet. 1998;352:291-292.

31. Claessens YE, Cariou A, Monchi M, et al. Detecting life-threatening lactic acidosis related to nucleoside-analog treatment of human immunodeficiency virus-infected patients, and treatment with L-carnitine. Crit Care Med. 2002;31:1042-1047. 
HIV/AIDS - Research and Palliative Care

Dovepress

\section{Publish your work in this journal}

HIV/AIDS - Research and Palliative Care is an international, peerreviewed open-access journal focusing on advances in research in HIV, its clinical progression and management options including antivira treatment, palliative care and public healthcare policies to control viral spread. The journal welcomes original research, basic science, clinical \& epidemiological studies, reviews \& evaluations, expert opinion \& commentary, case reports \& extended reports. The manuscript management system is completely online and includes a very quick and fair peer-review system. Visit http://www.dovepress.com/ testimonials.php to read real quotes from published authors.

Submit your manuscript here: http://www.dovepress.com/hivaids---research-and-palliative-care-journal 